\title{
Novel Compound Enables High-Level Adenovirus Transduction in the Absence of an Adenovirus-Specific Receptor
}

\author{
CHRISTINE M. FOULETIER-DILLING, ${ }^{1}, *$ PABLO BOSCH, ${ }^{2}, *$ ALAN R. DAVIS, $, 1,3,4$ \\ JESSICA A. SHAFER, ${ }^{1,3}$ STEVEN L. STICE, ${ }^{2}$ ZBIGNIEW GUGALA, ${ }^{4}$ FRANCIS H. GANNON, ${ }^{5}$ \\ and ELIZABETH A. OLMSTED-DAVIS ${ }^{1,3,4}$
}

\begin{abstract}
Viral vectors are extensively used to deliver foreign DNA to cells for applications ranging from basic research to potential clinical therapies. A limiting step in this process is virus uptake and internalization into the target cells, which is mediated by membrane receptors. Although it is possible to modify viral capsid proteins to target the viruses, such procedures are complex and often unsuccessful. Here we present a rapid, inexpensive system for improving transduction of cells, including those that lack receptors for adenovirus fiber proteins. Addition of GeneJammer (Stratagene, La Jolla, CA) during the adenovirus transduction led to a significant increase in both the total number of transduced cells and the level of transgene expression per cell. Studies using cell lines deficient in adenovirus receptors demonstrated that addition of GeneJammer provided a novel cellular entry mechanism for the virus. These findings were tested in a cell-based gene therapy system for the induction of bone, which is contingent on high-level expression of the transgene. Inclusion of GeneJammer in either Ad5BMP2 or Ad5F35BMP2 transduction of a variety of cells demonstrated a correlating increase in bone formation. The results suggest a novel and versatile method for achieving high-level transduction using adenovirus.
\end{abstract}

\section{OVERVIEW SUMMARY}

Adenovirus vectors are useful in gene transfer, ranking second behind DNA transfection as a DNA delivery method. However, host range and tissue tropism restrict the use of specific adenovirus vectors, sometimes necessitating the time-consuming construction of new vectors with more appropriate cell specificity. This is particularly important in bone formation using Ad5BMP2, where inefficient transduction can result in the total lack of bone formation. We describe here a novel methodology, using GeneJammer, that increases the transduction efficiency of adenovirus vectors in general in a receptor-independent fashion. We show that the use of this compound enables bone to be formed readily in vivo in immunocompetent animals.

\section{INTRODUCTION}

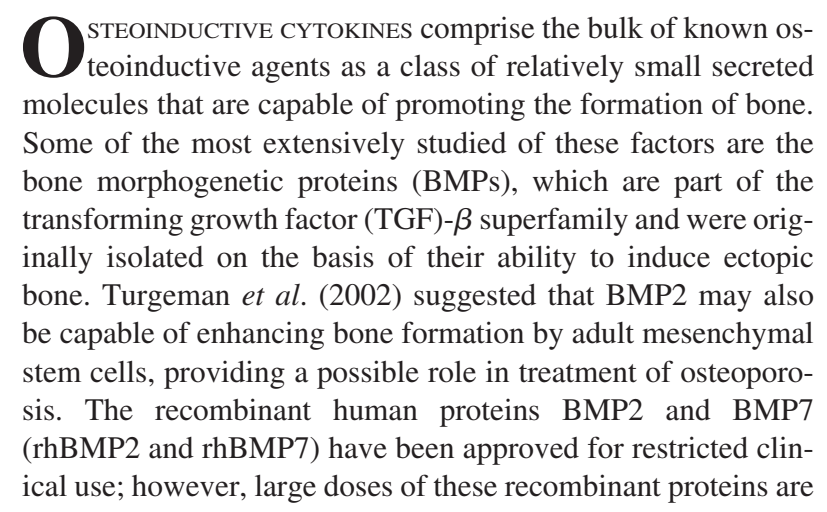

$\mathbf{O}$ EOINDUCTIVE CYTOKINES comprise the bulk of known osoinductive agents as a class of relatively small secreted molecules that are capable of promoting the formation of bone. Some of the most extensively studied of these factors are the bone morphogenetic proteins (BMPs), which are part of the transforming growth factor (TGF) $-\beta$ superfamily and were originally isolated on the basis of their ability to induce ectopic bone. Turgeman et al. (2002) suggested that BMP2 may also be capable of enhancing bone formation by adult mesenchymal stem cells, providing a possible role in treatment of osteoporosis. The recombinant human proteins BMP2 and BMP7 ical use; however, large doses of these recombinant proteins are

\footnotetext{
${ }^{1}$ Center for Cell and Gene Therapy, Baylor College of Medicine, Houston, TX 77030.

${ }^{2}$ Department of Animal and Dairy Science, University of Georgia, Athens, GA 30602.

${ }^{3}$ Department of Pediatrics, Baylor College of Medicine, Houston, TX 77030.

${ }^{4}$ Department of Orthopedic Surgery, Baylor College of Medicine, Houston, TX 77030

${ }^{5}$ Department of Bone Pathology, Armed Forces Institute of Pathology, Washington, D.C. 20306.

*C.M.F.-D. and P.B. contributed equally to this work.
} 
required to induce adequate bone repair, suggesting that the mode of BMP delivery still requires further optimization. Furthermore, BMP-induced osteogenesis, even under ideal conditions, does not approach the efficiency of bone formation seen during normal fracture repair (Franceschi et al., 2004). Also, delivery of these proteins requires a carrier such as collagen sponge carriers (Bonadio et al., 1999), which can also elicit responses that often influence the bone formation process.

Alternatively, gene therapy approaches have been developed to circumvent the problems associated with delivering the protein itself (Musgrave et al., 2000; Olmsted-Davis et al., 2002). Studies using both nonviral and viral delivery systems have successfully induced bone formation in several animal models. Park et al. (2003) used liposomes to transfect mesenchymal stem cells to heal mandibular defects in rats by an ex vivo strategy that showed markers of new bone matrix such as osteopontin and osteocalcin within 2 weeks of gene transfer. Musgrave et al. (2000) have shown that primary bone marrow mesenchymal stem cells (hBM-MSCs) transduced with Ad5BMP2 produced no radiographic ossification. We demonstrated (Olmsted et al., 2001) that adenovirus serotype 5 (Ad5) elicits only minimal amounts of BMP2 synthesis on transduction of hBM-MSCs because of the lack of adenovirus-specific receptors. Further, we previously demonstrated a critical link between the amount of bone morphogenetic protein- 2 expressed in the tissue and the production of bone (Gugala et al., 2003). Therefore the poor efficiency of delivery and transient expression of the gene are significant challenges that need to be overcome for these strategies to be truly effective.

Consequently, several approaches have been undertaken in order to circumvent these problems. A limiting step in adenoviral uptake is indeed the entry into target cells, which is mediated by the coxsackievirus-adenovirus receptor (CAR) and cellular $\alpha_{\mathrm{v}} \beta$ integrins. In many cell types, these receptors are either absent or expressed at low levels, leading to poor transduction efficiency by adenovirus. For instance, CAR expression is highly variable and most stem cells and fibroblast-type cells are poorly infected by adenoviral vectors because of low expression of CAR (Hidaka et al., 1999; Sakurai et al., 2003).

Investigators have attempted to circumvent this problem by introducing genetic fiber modifications in Ad, which provides an alternative cellular entry route. Chimeric Ad5 vectors possessing fiber proteins derived from subgroup B Ad serotypes such as Ad35 have become increasingly popular as gene transfer vectors because they can efficiently deliver genes to cell types that are refractory to Ad5 infection (Mizuguchi and Hayakawa, 2002; Olmsted-Davis et al., 2002; Gao et al., 2003). Group B adenoviruses have been shown to use CD46 as a primary attachment receptor (Gaggar et al., 2003; Segerman et al., 2003). However, receptors for these chimeric viruses appear to be absent on most murine cells (Mallam et al., 2004), which reduces their versatility, and high titers of the purified fibermodified viruses are difficult to produce.

Polycations and cationic lipids, which form complexes with adenoviral particles, have also been used to facilitate in vitro transduction of a range of cell types (Lanuti et al., 1999; Toyoda et al., 2001). Similarly, adenoviral infection of primitive human hematopoietic cells can be strongly enhanced by several cationic lipids (Harrison et al., 1995; Byk et al., 1998; Marit et al., 2000). Others have used bilamellar cationic liposomes to protect adenovectors from preexisting humoral immune responses (Yotnda et al., 2002).

The aim of this study was to investigate the role of a polyamine in enhancement of adenoviral transduction of several cell types in vitro, using a proprietary formulation of polyamine and other components in $80 \%$ ethanol (GeneJammer; Stratagene, La Jolla, CA). We show a significant increase in both the total number of transduced cells and the level of transgene expression per cell, when the transduction is carried out in the presence of GeneJammer. Studies using cell lines deficient in adenovirus receptors demonstrated that addition of GeneJammer provided a novel cellular entry mechanism for the virus. We further tested this methodology in our cell-based gene therapy system for induction of bone formation. The compound increased cell transduction and achieved a high level of functional BMP2 protein expression, in both murine and human cell types. Further, this increase in BMP2 production correlated with increased bone formation in vivo. This finding greatly advances our goal of developing a cell-based gene therapy system for the induction of bone formation. GeneJammer transfection reagent markedly increases the efficiency of adenovirus-mediated gene transfer in cells deficient in adenovirus receptors through an alternative pathway for virus entry into the cell. Hence this methodology provides a rapid, inexpensive technique for transduction of adenovirus-refractive cells.

\section{MATERIALS AND METHODS}

\section{Cell culture}

Cell lines. Chinese hamster ovary (CHO) cells, a human lung carcinoma cell line (A549), and a murine osteoblast cell line (MC3T3-E1) were obtained from American Type Culture Collection (Manassas, VA). Human lung carcinoma cells (A549) were propagated in Dulbecco's modified Eagle's medium (DMEM; Cambrex Bio Science Walkersville, Walkersville, MD) supplemented with $10 \%$ fetal bovine serum (FBS; HyClone, Logan, UT), penicillin (100 units/ml), streptomycin (100 $\mu \mathrm{g} / \mathrm{ml})$, and amphotericin B $(0.25 \mu \mathrm{g} / \mathrm{ml}$; Invitrogen, Gaithersburg, MD); CHO cells and MC3T3-E1 cells were propagated in RPMI and $\alpha$-MEM, respectively, supplemented as described above.

The W20-17 mouse stromal cell line (a gift from Genetics Institute, Cambridge, MA) was propagated as described by Thies et al. (1992). Briefly, the cells were grown in DMEM supplemented as described above and cultured at a subconfluent density in order to maintain the phenotype. All cell types were grown at $37^{\circ} \mathrm{C}$ and $5 \% \mathrm{CO}_{2}$ in humidified air.

Human bone marrow mesenchymal stem cells. Discarded human bone marrow products were obtained from healthy donors in compliance with all state and federal regulations, with institutional review board approval. Mononuclear cells were isolated by gradient density centrifugation on Ficoll-Paque PLUS (Amersham Biosciences/GE Healthcare, Piscataway, NJ) and washed twice with Dulbecco's phosphate-buffered saline (PBS; Invitrogen) before culturing. Cells were plated at a density of $5 \times 10^{6}$ cells $/ \mathrm{cm}^{2}$ (Jaiswal et al., 1997) in DMEM supplemented with $10 \%$ FBS and antibiotics-antimycotics as de- 
scribed above. Early adherent fibroblastic cells appeared within 2 days of culture, and after 1 week dead cells and debris were removed by washing with PBS and cells were passaged before confluence. Several vials of these cells were frozen in Origen dimethyl sulfoxide freeze medium (Igen International, Gaithersburg, MD). Where indicated, hBM-MSCs were treated by addition of $1 \mu M$ dexamethasone (Sigma, St. Louis, MO) to the culture medium.

\section{Transduction of cells with adenovirus in the presence or absence of GeneJammer}

Adenoviruses. Replication-defective E1- and E3-deleted first-generation human Ad5 and/or modified forms in which the normal fiber protein has been substituted for the human adenovirus type 35 fiber (Ad5F35) were constructed to carry cDNAs for either BMP2 or green fluorescent protein (GFP) in the E1 region of the virus (Olmsted et al., 2001). Ratios of virus particles (VP) to plaque-forming units (PFU) were 55, 76, 8, 132, and 200 for Ad5BMP2, Ad5F35BMP2, Ad5eGFP, Ad5F35eGFP, and Ad5-empty, respectively, and all viruses were shown to be negative for replication-competent adenovirus. The VP:PFU ratio corresponds to MOIs (plaque-forming units per cell) of $45,32,312,20$, of 12.5 , respectively.

Cell transduction. Adherent cells $\left(1 \times 10^{6}\right)$ were transduced with adenovirus at three different virus concentrations $(2500$, 5000 , and $10,000 \mathrm{VP} /$ cell) with or without $1.2 \%$ GeneJammer. This concentration of the polyamine compound was optimized on the basis of percentage of GFP transduction (data not shown). Briefly, $15 \mu \mathrm{l}$ of GeneJammer or PBS was added to $500 \mu \mathrm{l}$ of DMEM without supplements and incubated for 10 min at room temperature. The virus was then added at one of the indicated concentrations and the mixture was further incubated for $10 \mathrm{~min}$ at room temperature. This virus-GeneJammer mixture was added to approximately $1 \times 10^{6}$ cells along with $750 \mu \mathrm{l}$ of DMEM supplemented with $10 \%$ FBS and antibiotics-antimycotics. The cells were incubated at $37^{\circ} \mathrm{C}$ for $4 \mathrm{hr}$ and then the mixture was diluted with $3 \mathrm{ml}$ of fresh medium containing FBS.

\section{Flow cytometry}

Transduced cells were trypsinized, washed, and resuspended in PBS $48 \mathrm{hr}$ after the initial transduction. Dead cells and debris were excluded from analysis by inclusion of propidium iodide (PI). Flow cytometric analysis was performed with a FACSCalibur cytometer (BD Biosciences Immunocytometry Systems, San Jose, CA). The percentage of GFP-positive cells was quantified with CellQuest software (BD Biosciences Immunocytometry Systems), using a minimum of $10^{5}$ cells per sample $(n=3)$. Relative fluorescence intensity (RFI) of the GFP-positive cell population was also determined for each sample. All data were taken in triplicate and reported as means and standard deviation. Significance was determined between samples transduced with and without GeneJammer $(n=3)$, using a Student $t$ test with $95 \%$ confidence interval $(p<0.05)$ (InStat software; GraphPad Software, San Diego, CA).

A549 cells, hBM-MSCs, and CHO cells were analyzed for CAR, $\alpha_{\mathrm{v}}$ integrin, and CD46 expression. Briefly, the cells at a concentration of $1 \times 10^{6} / \mathrm{ml}$ were incubated with the primary antibody: CAR, a mouse anti-human antibody (ab9891; Abcam, Cambridge, UK), $\alpha_{\mathrm{v}}$ integrin, a mouse anti-human antibody (MAB1953Z; Chemicon International, Temecula, CA), or CD46, a mouse anti-human antibody (CBL488; Chemicon International) for $15 \mathrm{~min}$ on ice. The secondary antibody, fluorescein isothiocyanate (FITC)-conjugated rat anti-mouse (553354; BD Biosciences Pharmingen, San Diego, CA) was diluted 1:200 and cells were incubated with the secondary antibody for $15 \mathrm{~min}$ on ice. The cell pellet was washed in PBS and the samples were analyzed by flow cytometry.

\section{Quantification of BMP2}

BMP2 protein was measured in culture supernatant taken from cells $72 \mathrm{hr}$ after transduction with various concentrations of Ad5BMP2 in the presence or absence of GeneJammer. Briefly, $10^{6}$ cells were transduced as described above and culture supernatant was collected and assayed with a Quantikine BMP2 immunoassay (DBP200; R\&D Systems, Minneapolis, MN).

\section{Alkaline phosphatase assay}

W20-17 cells were assayed for alkaline phosphatase activity 3 days after addition of either Ad5BMP2, Ad5-empty, or medium, using a chemiluminescence procedure (Olmsted et al., 2001). Cellular alkaline phosphatase was extracted by three freeze-thaw cycles in a $100-\mu \mathrm{l} / \mathrm{cm}^{2}$ concentration of $25 \mathrm{~m} M$ Tris- $\mathrm{HCl}(\mathrm{pH} 8.0)$ and $0.5 \%$ Triton X-100 and activity was then measured by addition of CSPD substrate with Sapphire-II enhancer (Applied Biosystems, Foster City, CA) to the samples. The light output from each sample was integrated for $10 \mathrm{sec}$ (after a 2-sec delay) with a luminometer (TD-20/20; Turner Designs, Sunnyvale, CA). Alkaline phosphatase levels were recorded as relative luminescence units (RLU) and normalized to protein content with the bicinchoninic acid (BCA) assay (Pierce Biotechnology, Rockford, IL), using bovine serum albumin to derive a standard curve. Data are presented as percent induction relative to that of unstimulated basal control cells. Statistical analysis was performed as described previously. Briefly, all data were taken in triplicate and reported as means and standard deviation. A Student $t$ test with $95 \%$ confidence interval $(p<0.05)$ was done between the untreated control and each experimental condition.

\section{Heterotopic bone assay}

hBM-MSCs were transduced with Ad5BMP2 (2500 VP/cell) or Ad5-empty (2500 VP/cell), the latter being a control adenovirus type 5 vector that lacks a transgene in the E1-deleted region. Briefly, cells were removed with trypsin, resuspended at a concentration of $5 \times 10^{6}$ cells/100 $\mu \mathrm{l}$ of PBS, and then injected into the hindlimb quadriceps muscle of nonobese diabetic/severe combined immunodeficiency (NOD/SCID) mice (two animals per group). All animals were killed 2 weeks after injection and the hindlimbs were harvested, the skin removed, and the limbs placed in formalin. Bone formation was analyzed by X-ray analysis, using an MX-20 specimen radiography system model ( $83 \mathrm{sec}$ at $31 \mathrm{kV}$; Faxitron, Wheeling, IL). All animal studies were performed in accordance with standards of the Department of Comparative Medicine of Baylor College 
of Medicine (Houston, TX), after review and approval of the protocol by the Animal Use and Care Committee.

\section{RESULTS}

\section{Enhanced viral transduction in the presence of GeneJammer}

Adenovirus transduction of coxackie-adenovirus receptor (CAR)-negative cell lines is extremely inefficient, requiring large amounts of virus and resulting in low-level expression of the desired transgene. To enhance virus uptake into the cells, we tested the ability of the commercially available polyamine complex, GeneJammer, to enhance virus transduction. Accordingly, CAR-negative hBM-MSCs ( $10^{6}$ cells) were transduced with Ad5eGFP virus at three different concentrations (2500, 5000 , and 10,000 VP/cell) in the presence or absence of GeneJammer (Fig. 1A). Adenovirus type 5 lacking any transgene (Ad-empty) was used as a negative control. As seen in Fig. 1A, the presence of the polyamine GeneJammer increases the number of transduced hBM-MSCs at all concentrations of virus. The lowest MOI, $2500 \mathrm{VP} / \mathrm{cell}$, resulted in the most dramatic increase in number of transduced cells, with $15 \%$ in the absence of GeneJammer and $95 \%$ transduced in the presence of the polyamine. Further, we observed an increase in the number of transduced cells with a corresponding increase in virus MOI in the absence of GeneJammer; however, in the presence GeneJammer, the maximum number of transduced cells (95-100\%) was found for all virus concentrations.

Because multiple virus particles can enter the same cell, we also analyzed samples transduced in the presence of GeneJammer, and in which we obtained $95-100 \%$ cell transduction, to determine whether the intensity of GFP expression increased with virus concentration. As can be seen in Fig. 1B, the intensity of GFP expression from cells transduced with GeneJammer increased with virus dose. Because $100 \%$ of the cells were transduced at all virus doses in this population, the increase in GFP intensity presumably represents an increase in the number of virus particles each cell is taking up. The data suggest that GeneJammer not only enhances the number of cells taking up the virus (Fig. 1A) but also the total amount of virus entering any given cell (Fig. 1B).

\section{GeneJammer compound allows adenovirus to enter cells lacking the receptor for fiber}

Two potential models exist as to the mechanism by which the polyamine enhances virus uptake. First, GeneJammer may aid in virus binding to its receptor, therefore potentially acting as a coreceptor for the virus internalization. Alternatively, this compound may bind to the virus and promote a novel entry route into the cell. To determine which of these is most likely, we compared the transduction efficiency of various cell types known to have differential expression levels of CAR and $\alpha_{\mathrm{v}}$ integrin (Ad5) or CD46 (Ad5F35), in the presence or absence of GeneJammer. The three cells lines chosen have the following receptor characteristics: A549 cells express high levels of CAR, $\alpha_{\mathrm{v}}$ integrin, and CD46; hBM-MSCs lack CAR but express $\alpha_{\mathrm{v}}$ integrin, and express moderate levels of CD46; and $\mathrm{CHO}$ cells express few or no adenovirus receptors (Table 1). As expected,
Ad5eGFP (2500 VP/cell) transduced $100 \%$ of the receptor-positive A549 cells, whereas less than $15 \%$ of the receptor-negative $\mathrm{CHO}$ cells were transduced (Fig. 2A and C). hBM-MSCs, which are $\alpha_{\mathrm{v}}$ integrin positive and CAR negative, yielded approximately $10 \%$ of cells expressing GFP at this same MOI (Fig. 2B). Parallel transductions done in the presence of GeneJammer showed significant enhancement of transduction in cells lacking CAR but expressing $\alpha_{\mathrm{v}}$ integrin, as well as in receptor-negative cells (Fig. 2B and C). The results in Fig. 2C show that approximately $95 \%$ of transduced $\mathrm{CHO}$ cells expressed GFP when the adenovirus was delivered in the presence of the polyamine, suggesting that the molecular mechanism is not adenovirus receptor associated but, rather, a novel pathway for virus entry into the cells. We did not see any significant changes between the groups in A549 cells (Fig. 2A) because the cells in the absence of GeneJammer were maximally transduced. However, the data suggest that these two systems for virus entry do not appear to inhibit one another (Fig. 2A).

We next chose to determine whether virus entry into cells via the polyamine was specific to the adenovirus type 5 capsid, and therefore similar experiments were conducted with the altered fiber virus Ad5F35eGFP. Results were similar to those obtained with the Ad5eGFP vector, suggesting a similar mechanism that is not dependent on adenovirus type 5 fiber for entry into the cell (Fig. 2).

\section{BMP2 expression and bone formation in the presence of GeneJammer}

We previously reported the inability of Ad5BMP2 to transduce hBM-MSCs, which resulted in low-level expression of BMP2 and lack of bone formation in vivo (Olmsted-Davis et al., 2002). In addition, because eGFP has been engineered to be extremely stable, whereas BMP2 is significantly less stable at both the RNA and protein levels, enhancement by the polyamine may be underestimated when eGFP is used as a transgene. To determine this, we measured the amount of BMP2 secreted into the culture supernatant after transduction of hBMMSCs with Ad5BMP2, in the presence or absence of GeneJammer. Expression and secretion of BMP2 in hBM-MSCs were enhanced after combined GeneJammer-Ad5BMP2 transduction. Culture supernatants were collected $72 \mathrm{hr}$ after initial transduction and BMP2 was quantified by enzyme-linked immunosorbent assay (ELISA). The results (Fig. 3A) show increased BMP2 protein in culture supernatant taken from $\mathrm{hBM}$ MSCs transduced in the presence of the polyamine compared with hBM-MSC cultures from which GeneJammer was omitted during transduction. BMP2 was not detected in the culture supernatant from either cells alone or those transduced with the control virus Ad-empty, demonstrating that BMP2 expression in all cases specifically corresponded with viral transduction (Fig. 3A).

BMP2 protein in culture supernatant was further assayed for functional activity, using the W20-17 cell-based assay (Thies et al., 1992; Blum et al., 2001; Olmsted et al., 2001). In this assay, the murine bone marrow cell line W20-17 was exposed to culture supernatant from hBM-MSCs that had been transduced in the presence or absence of GeneJammer. This cell line responds to exogenously added BMP2 by increasing the expression of alkaline phosphatase. Culture supernatants from 
A

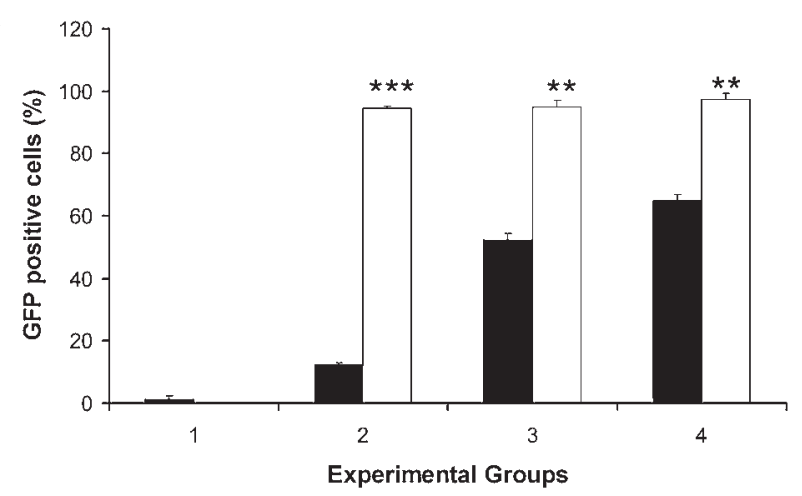

FIG. 1. (A) Flow cytometric quantification of GFP expression after transduction of hBM-MSCs with (1) Ad5-empty, (2) Ad5eGFP at $2500 \mathrm{VP} / \mathrm{cell}$, (3) Ad5eGFP at $5000 \mathrm{VP} / \mathrm{cell}$, or (4) Ad5eGFP at 10,000 VP/cell in the absence (solid columns) or presence (open columns) of GeneJammer. The percentage of GFP-positive cells is depicted as the average eGFP fluorescence, where $n=3$. Columns and error bars represent means \pm standard deviation, respectively, for $n=3$ experiments. $* * * p<$ 0.001 and $* * p<0.01$ (Student $t$ test). (B) GFP fluorescence intensity shifts in the flow cytometry profiles of eGFP expression in hBM-MSCs transduced with Ad5GFP at either 2500, 5000, or 10,000 VP/cell, in the presence or absence of GeneJammer [as shown in (A)]. In all samples 100\% of the cells were found to express eGFP.
B
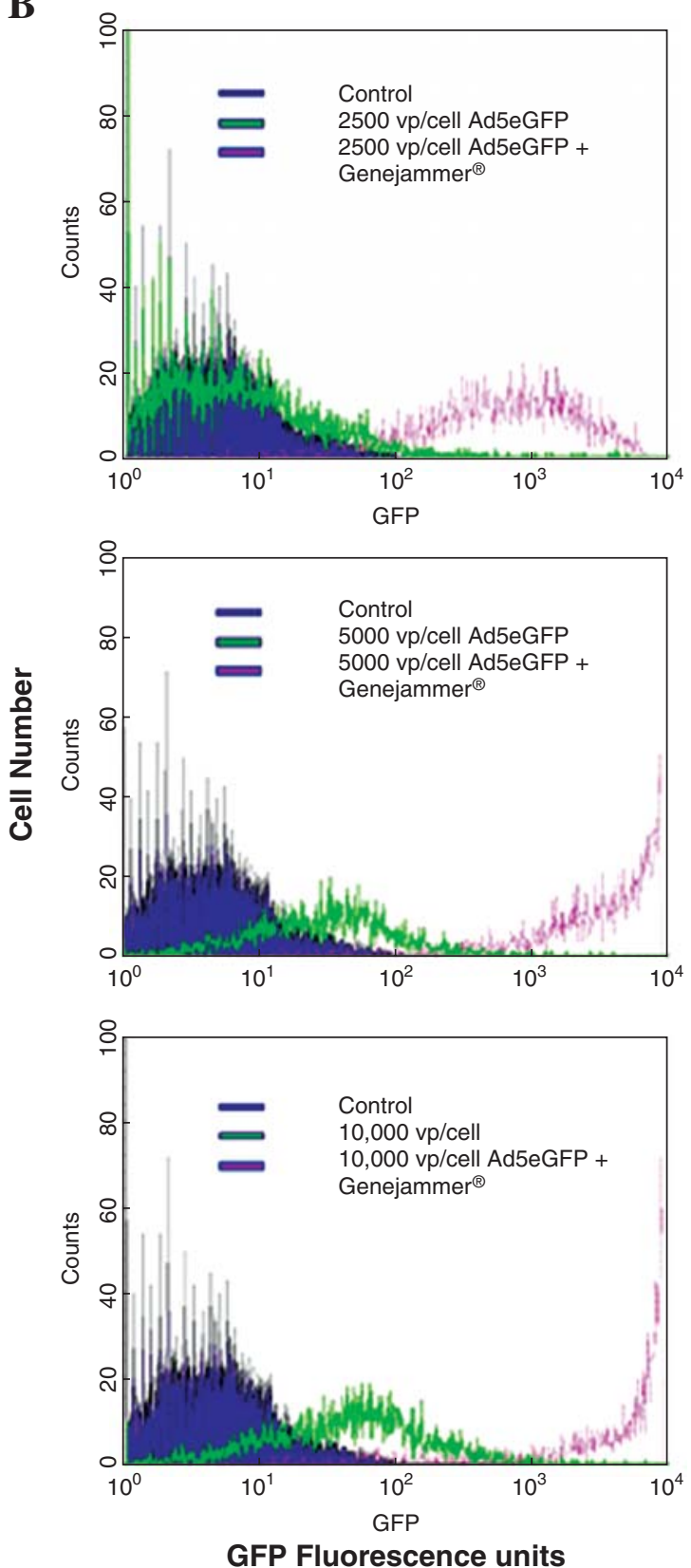

duced in the absence of the polyamine appeared to increase with viral dose (Fig. 3B).

We have previously shown that transduction of hBM-MSCs with Ad5BMP2 induces little to no detectable bone formation whereas those cells transduced with Ad5F35BMP2 elicited significant bone formation in the same 2-week time period, suggesting that efficiency of cell transduction was critical to bone formation in vivo (Olmsted-Davis et al., 2002). To further confirm that GeneJammer can significantly increase Ad5BMP2 transduction efficiency and BMP2 production in hBM-MSCs without altering cell viability in vivo, we chose to compare these two cell populations in our heterotopic bone assay (OlmstedDavis et al., 2002). Briefly, hBM-MSCs $\left(5 \times 10^{6}\right)$ transduced with either Ad5BMP2 or the control vector Ad5-empty in the 
Table 1. Adenovirus Receptor Expression on Selected Cell Lines ${ }^{\mathrm{a}}$

\begin{tabular}{lccr}
\hline \multirow{2}{*}{ Cell line } & \multicolumn{3}{c}{ Expression (\%) of cell surface receptor } \\
\cline { 2 - 4 } & CAR & Integrin $\alpha_{v}$ & \multicolumn{2}{c}{ CD46 } \\
\hline A549 & $93.28 \pm 1.28$ & $99.26 \pm 0.53$ & $99.27 \pm 0.79$ \\
hBM-MSCs & $0.34 \pm 0.50^{\mathrm{b}}$ & $87 \pm 4.70^{\mathrm{b}}$ & $31.78 \pm 3.54$ \\
CHO & $1.77 \pm 0.27$ & $2.04 \pm 0.16$ & $0.19 \pm 0.05$ \\
\hline
\end{tabular}

Abbreviations: CAR, coxsackievirus-adenovirus receptor; $\mathrm{CHO}$, Chinese hamster ovary; hBM-MSCs, human bone marrow mesenchymal stem cells.

${ }^{a}$ Numbers represent the mean percentage of cells expressing each receptor \pm $\mathrm{SD}(n=3)$.

${ }^{\text {b}}$ From Olmsted-David et al. (2002).

presence or absence of GeneJammer were injected into the hindlimb quadriceps muscle of NOD/SCID mice. After 2 weeks, the injected tissues were isolated and bone formation was determined by radiological analysis. As can be seen in Fig. 3C, mice that received cells transduced with Ad5BMP2 in the presence of GeneJammer had detectable mineralized bone, whereas those that received cells transduced in the absence of the polyamine had none. In no cases did we detect bone in mice receiving cells transduced with the Ad-empty control vector (data not shown). The data suggest that bone formation correlated with enhanced transduction efficiency and expression of BMP2.

We next determined the effect of GeneJammer on adenovirus transduction of the murine cell line MC3T3-E1. MC3T3$\mathrm{E} 1$ is a $\mathrm{C} 57 \mathrm{BL} / 6$-derived cell line that can be propagated in vitro, and used in vivo in C57BL/6 mice without eliciting a graft-versus-host response in the animals. However, they do not possess CAR or CD46 (data not shown), and therefore are not readily transduced with $\mathrm{Ad} 5$ or $\mathrm{Ad} 5 \mathrm{~F} 35$ vectors. To overcome this barrier the cells were transduced with Ad5BMP2 at various concentrations $(2500,5000$, and $10,000 \mathrm{VP} /$ cell) in the presence or absence of GeneJammer. Culture supernatant was removed approximately $72 \mathrm{hr}$ after transduction and assayed for functional BMP2 activity, using W20-17 cells. Supernatant from cells transduced with Ad5BMP2 at $5000 \mathrm{VP} /$ cell, in the presence of GeneJammer, yielded a significant elevation (approximately 2.5 -fold) in alkaline phosphatase activity as compared with similar amounts of culture supernatant taken from parallel samples of cells transduced in the absence of GeneJammer (Fig. 4A). Further, the level of alkaline phosphatase induction was similar to that obtained with culture supernatant removed from hBM-MSCs after transduction with Ad5F35BMP2 (Fig. 4A).

We have observed that we can make copious amounts of bone in immunodeficient NOD/SCID mice (Olmsted-Davis et al, 2002; Gugala et al, 2003) on injection of various human cell types transduced with AD5F35BMP2. However, in our current investigations we wished to inject transduced cells into immunocompetent mice. To aid in this process we transduced and injected murine cells (MC3T3-E1) rather than human cells. Approximately $5 \times 10^{6} \mathrm{MC} 3 \mathrm{~T} 3$-E1 cells were transduced with either Ad5BMP2 (5000 VP/cell) or Ad5-empty (5000 VP/cell) in the presence or absence of GeneJammer and injected into the hindlimb quadriceps muscle of C57BL/6 mice $(n=4)$. Figure 4B shows the results of radiological analysis of the hindlimbs of only two of the mice, because all mice gave identical results, approximately 2 weeks after injection of the transduced cells.
Mice that received cells transduced with Ad5BMP2 in the presence of GeneJammer displayed detectable mineralization whereas cells transduced in the absence of the polyamine showed little to no detectable bone. These findings demonstrate the ability of BMP2-transduced cells to elicit bone formation
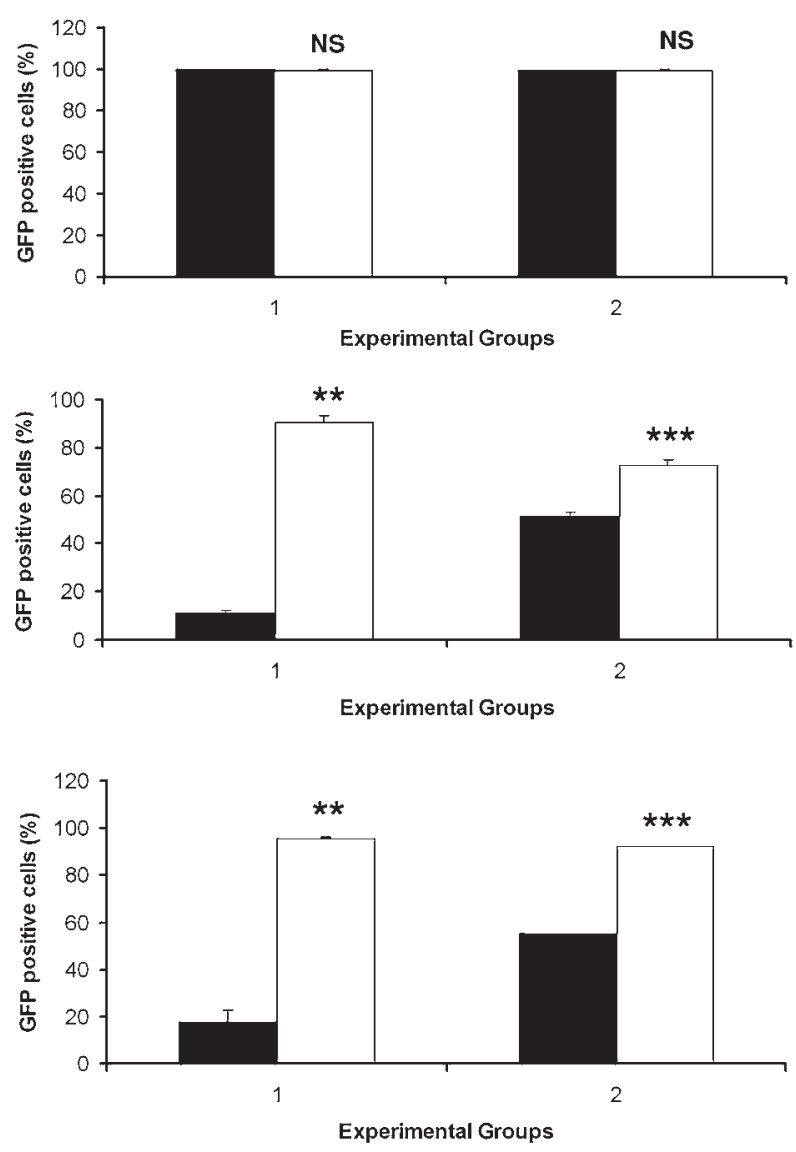

FIG. 2. Flow cytometry analysis of GFP expression of A549 cells (A), hBM-MSCs (B), and CHO cells $(\mathbf{C})$ transduced with Ad5eGFP at $2500 \mathrm{VP} /$ cell (group 1) or with Ad5F35eGFP at $2500 \mathrm{VP} /$ cell (group 2) in the absence (solid columns) or presence (open columns) of GeneJammer. The percentage of GFPpositive cells is depicted as the average GFP fluorescence, where $n=3$. Columns and error bars represent means \pm standard deviation, respectively, for $n=3$ experiments. $* * * p<$ 0.001 and $* * p<0.01$ (Student $t$ test). 
A

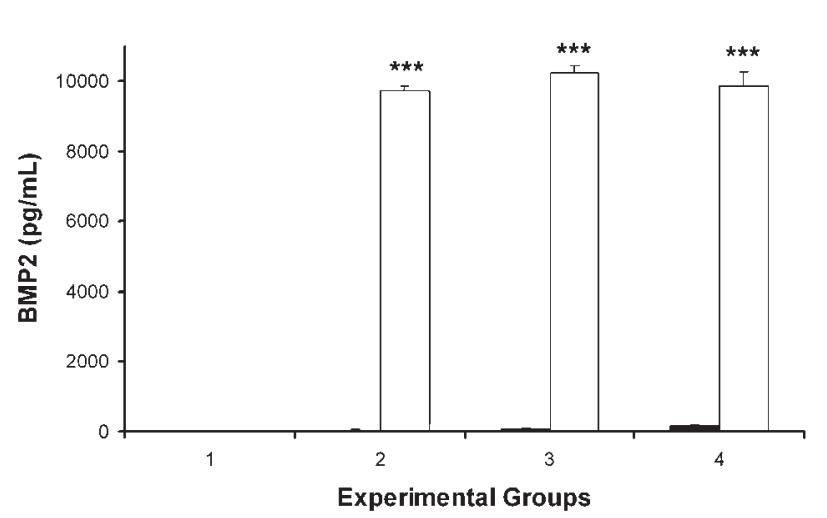

C

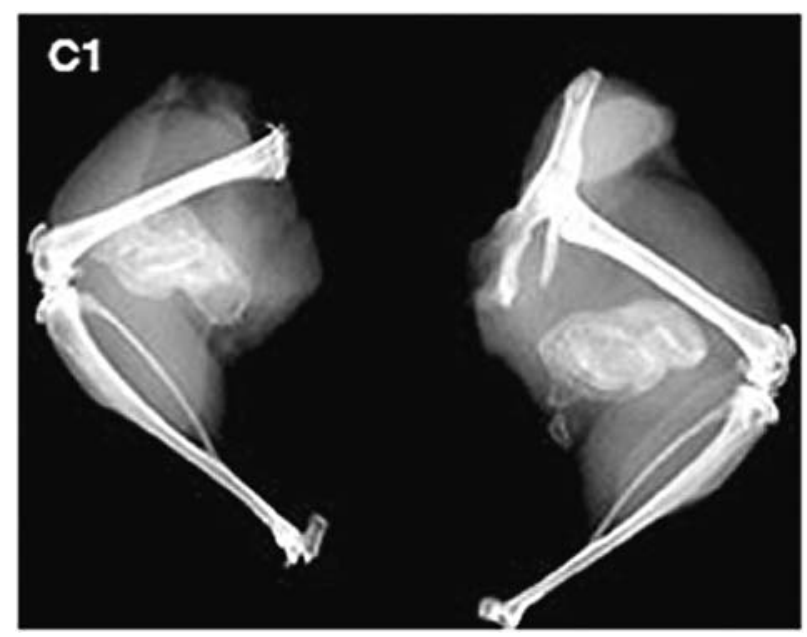

B
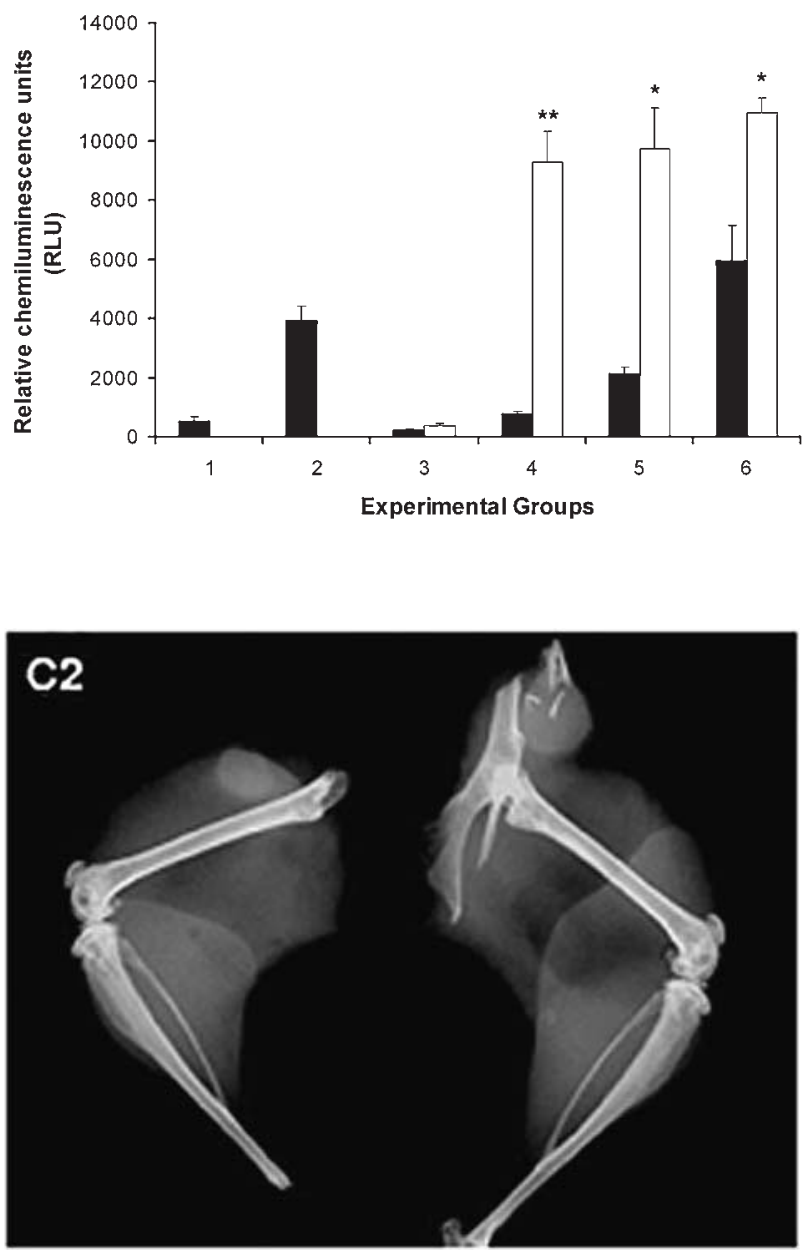

FIG. 3. Quantification of BMP2 protein (A), and activity in vitro (B) or in vivo (C). (A) BMP2 protein concentration was determined in culture supernatants, using the Quantikine BMP2 assay (R\&D Systems). BMP2 activity was measured in culture supernatant taken from hBM-MSCs alone (group 1) and from hBM-MSCs transduced with Ad5BMP2 at $2500 \mathrm{VP} / \mathrm{cell}$ (group 2), $5000 \mathrm{VP} /$ cell (group 3), and 10,000 VP/cell (group 4) in the absence (solid columns) or presence (open columns) of GeneJammer. The concentration of BMP2 was extrapolated from a standard curve based on known concentrations of recombinant BMP2. BMP2 concentrations in the supernatant are reported as picograms per milliliter, $n=3$. *** $p<0.001$ (Student $t$ test). (B) BMP2 activity was measured in culture supernatant taken from hBM-MSCs alone (group 1), from hBM-MSCs transduced with control Ad5F35BMP2 (2500 VP/cell) (group 2), from hBM-MSCs transduced with Ad5-empty (5000 VP/cell) (group 3), or from hBM-MSCs transduced with Ad5BMP2 at $2500 \mathrm{VP} /$ cell (group 4), $5000 \mathrm{VP} /$ cell (group 5), or 10,000 VP/cell (group 6) by determining the increase in alkaline phosphatase activity in W20-17 cells $72 \mathrm{hr}$ after exposure, in the absence (solid columns) or presence (open columns) of GeneJammer. Alkaline phosphatase activity is depicted as average relative chemiluminescence units (RLU), where $n=3$. Columns and error bars represent means \pm standard deviation, respectively, for $n=3$ experiments. $* * p<0.01$ and $* p<0.05$ (Student $t$ test). (C) Radiologic analysis of heterotopic bone formation in NOD/SCID mice after intramuscular injection of hBM-MSCs transduced with Ad5BMP2 (5000 VP/cell) plus GeneJammer (panel C1) or Ad5BMP2 alone (panel C2).

even in mice with a normal immune system. Further, the results suggest once again that the level of Ad5BMP2 transduction of the cells is crucial to elicit bone formation.

\section{DISCUSSION}

Much emphasis in tissue engineering of bone has been focused on developing gene therapy approaches to induce bone formation. However, many critical obstacles still stand in the pathway of developing such a system for clinical use, such as efficient transduction of either delivery cells or target tissues, sequestration of the cell or vector system to the specific site, and avoidance of immune surveillance and clearing before obtaining the desired bone induction.

We and others have taken a virus-based gene therapy approach to deliver BMP2 ectopically for induction of new bone formation (Olmsted-Davis et al., 2002; Gugala et al., 2003; Tsuda et al., 2003; Zhu et al., 2004). Because BMP2 is a secreted protein, the transduced cells function as a delivery vehi- 


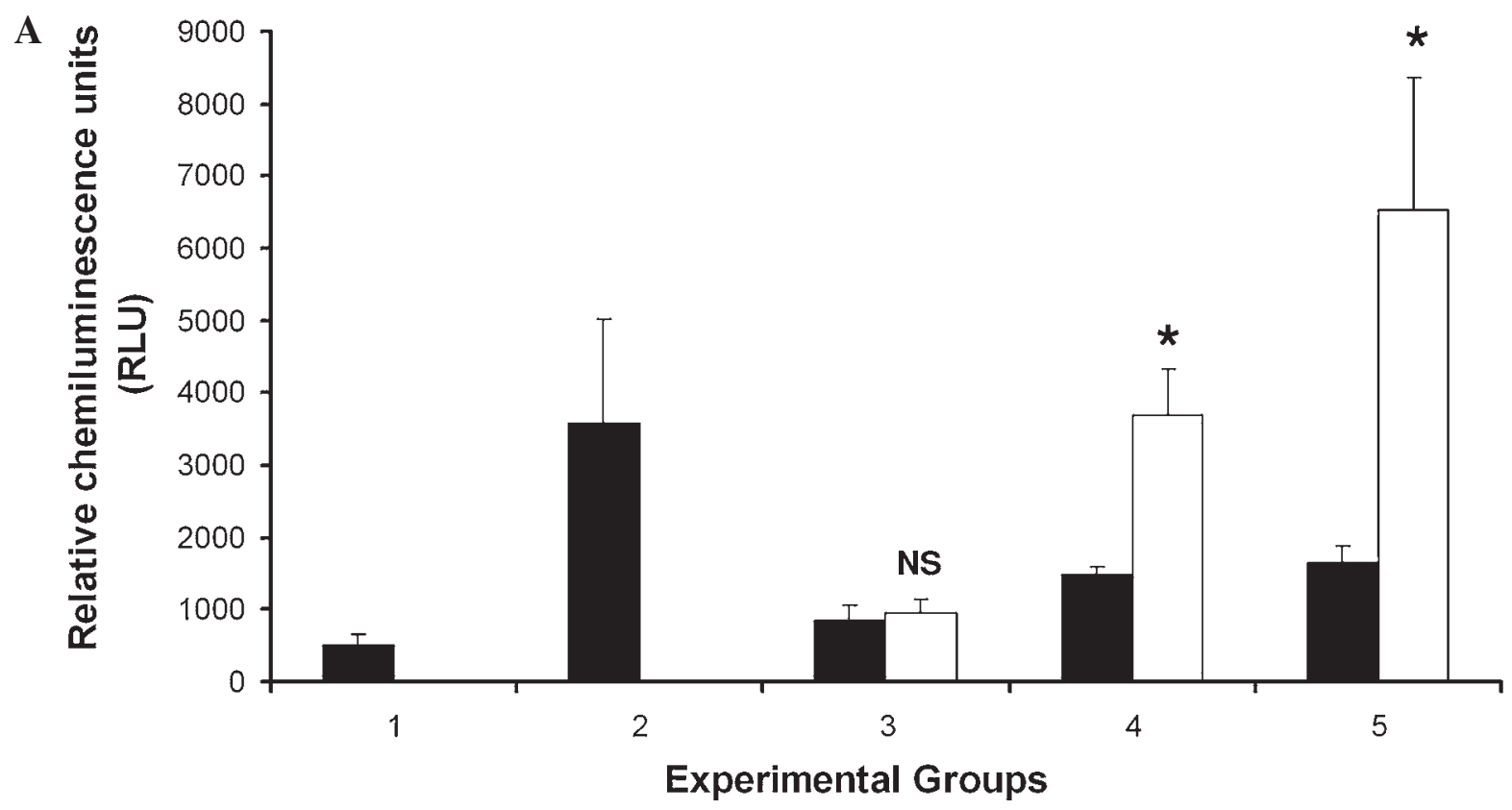

\section{B}
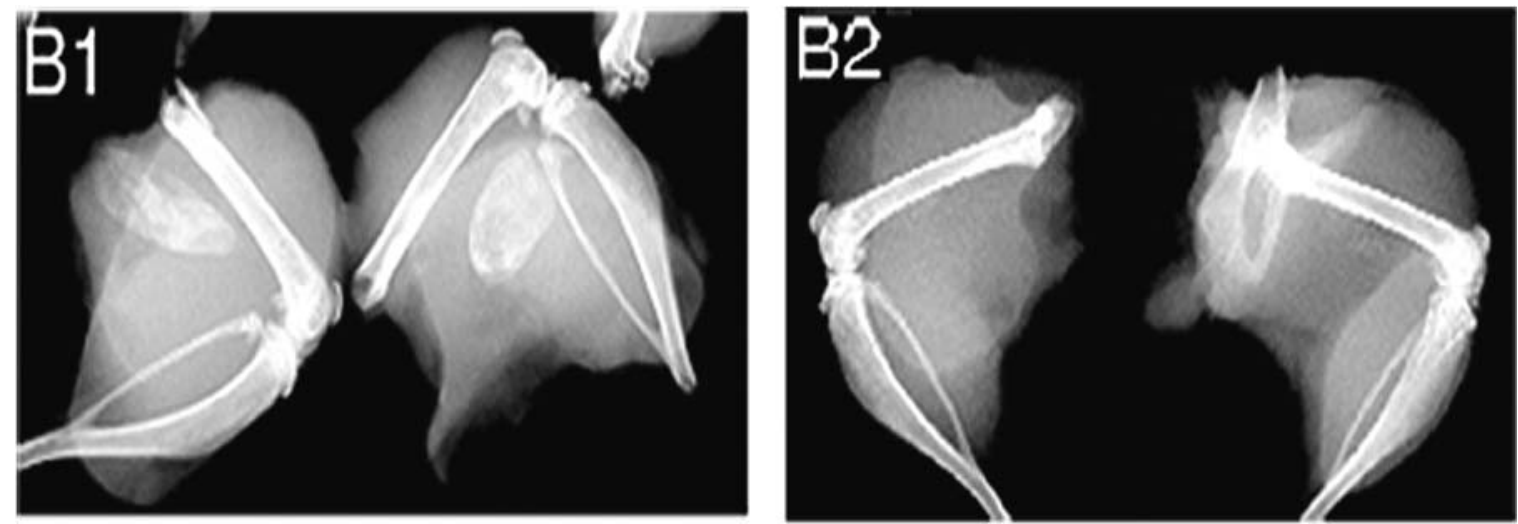

FIG. 4. Analysis of BMP2 activity both in vitro and in vivo. (A) BMP2 activity was analyzed in culture supernatant taken from MC3T3-E1 cells (group 1), from MC3T3-E1 cells transduced with Ad5F35BMP2 (2500 VP/cell) (group 2), and from MC3T3E1 cells transduced with Ad5BMP2 at $2500 \mathrm{VP} /$ cell (group 3), $5000 \mathrm{VP} /$ cell (group 4), or 10,000 VP/cell (group 5), in the absence (solid columns) or presence (open columns) of GeneJammer. Alkaline phosphatase activity is depicted as average relative chemiluminescence (RLU), where $n=3$. Columns and error bars represent means \pm standard deviation, respectively, for $n=3$ experiments. ${ }^{*} p<0.05$ (Student $t$ test). (B) In vivo analysis of BMP2 activity was determined radiologically in C57BL/6 mice that received MC3T3-E1 cells transduced with Ad5BMP2 (5000 VP/cell) in the presence (panel B1) or absence (panel B2) of GeneJammer.

cle (Gugala et al., 2003), but they provide additional safety because no free virus is delivered to patients, which reduces both immune clearance and acute reaction to the virus itself. Ideally these cells would be readily obtainable from patients, such as from the peripheral blood, which would avoid additional immune response to foreign cells. However, we have previously demonstrated that bone formation was linked to the level of BMP2 secretion, with high levels of BMP2 inducing more bone formation than low levels. Hence transduction methodology must be versatile enough to provide high-level transduction of a variety of species required for preclinical development, as well as the cell types found in human blood.
Here we have presented a rapid, easy, inexpensive, and versatile method for achieving high-level adenovirus transduction of cells from various species, by inclusion of the DNA transfection reagent GeneJammer. Comparison of eGFP expression after transduction with Ad5eGFP or Ad5F35eGFP showed a significant increase in both the number of cells and the intensity of transgene expression in the presence of GeneJammer, independent of fiber type. Interestingly, in experiments in which $100 \%$ of the cells were transduced at all viral doses in the presence of GeneJammer, we observed an increase in GFP intensity corresponding with increasing amounts of adenovirus. As can be seen in Fig. 1B, at 2500 VP/cell, $100 \%$ of the cells are 
transduced. However, the mean intensity of eGFP expression is approximately $10^{3}$ eGFP fluorescence units, whereas at 5000 $\mathrm{VP} /$ cell the mean is greater than $10^{4} \mathrm{eGFP}$ fluorescence units. Further, at the highest virus concentration $(10,000 \mathrm{VP} / \mathrm{cell})$ approximately $60 \%$ of the cells express $10^{4}$ eGFP fluorescence units, or more, as compared with the lower dose (5000 VP/cell), at which only $30 \%$ of the cells express this level of eGFP intensity. These results suggest that the mechanism by which GeneJammer assists adenovirus entry into cells is not limiting at the doses studied and that it can also increase the total number of adenovirus particles entering any individual cell, resulting in higher levels of expression per cell.

Because adenovirus fiber protein is involved in receptor binding and internalization, two likely models exist as to how the compound enhances cellular transduction. First, GeneJammer may bind the virus and allow cellular entry through either an alternative receptor or a non-receptor-mediated pathway. Alternatively, the compound may enhance adenovirus binding to its normal receptor, functioning as a coreceptor.

To determine which of the two tentative mechanisms was involved, we selected three different cell lines that had various levels of adenovirus receptors and transduced them in the presence or absence of GeneJammer. As expected, Ad5eGFP was able to infect $100 \%$ of the CAR-positive cell line A549, but was less efficient at transducing hBM-MSCs (which possess only the $\alpha_{\mathrm{v}}$ receptor), with $10 \%$ of the cells positive for eGFP expression. Further, in $\mathrm{CHO}$ cells, which are negative for both adenovirus-specific receptors, we observed no cells expressing eGFP. However, when GeneJammer was included in the transduction reaction, approximately $100 \%$ of all three cell types expressed the transgene. Significant enhancement of expression was achieved in the cell lines that lacked one or both adenovirus type 5-specific receptors, whereas in the receptor-positive cell line the transduction efficiency remained the same, presumably because it was maximal under both circumstances. Interestingly, the results suggest that GeneJammer is not enhancing viral entry through its normal receptor/coreceptormediated route but, rather, is using a novel mechanism, because $100 \%$ of the cells lacking adenovirus type 5 -specific receptors were transduced. This finding is consistent with other studies in which uptake of adenovirus via hexon protein binding to dipalmitoyl phosphatidylcholine resulted in cellular internalization via an alternative mechanism that does not use receptor binding (Balakireva et al., 2003). However, the authors suggested that this is a mechanism used by adenovirus for entry into the lung, because alveolar epithelial type II cells synthesize the major component of pulmonary surfactant, disaturated phosphatidylcholine, on adenovirus infection. Further, they suggested that although the cells do possess adenovirus-specific receptors, it is the production of this compound that leads to the high transduction efficiency found with these cell types, including A549 cells (Balakireva et al., 2003). Further studies are required to determine whether GeneJammer functions through similar mechanisms.

GeneJammer significantly enhanced expression of eGFP when Ad5F35eGFP was used in the transductions. Again, this suggests that the mechanism by which GeneJammer enhances virus entry into the cell is not specific to fiber type 5. As expected, $100 \%$ of A549 cells, which possess the adenovirus type 35 -specific receptor CD46, were transduced in the presence or absence of the compound. Interestingly, we observed approximately the same transduction efficiency with both hBM-MSCs and $\mathrm{CHO}$ cells when using Ad5F35eGFP, even though these cell lines differ significantly in the level of CD46. One possible explanation is that the anti-human CD46 antibody used in these experiments may not detect hamster CD46. The lower level of induction by GeneJammer is in part due to the higher transduction efficiency of the Ad5F35eGFP vector in its absence, with approximately $55-60 \%$ of the cells transduced. Ad5eGFP transduction of CHO cells, however, is low in the absence of GeneJammer, and it has been shown previously (Bergelson et al., 1997; Davison et al., 1999) that CHO cells lack CAR, because CHO cells can be transduced by Ad5 vectors when these cells are transfected with a vector that elicits the expression of CAR.

We next measured the level of BMP2 production in cells transduced with Ad5BMP2 to determine whether GeneJammer could enhance the expression of this protein. As seen in Fig. $3 \mathrm{~A}-\mathrm{C}$, there was a significant enhancement in BMP2 production and activity in vitro and in vivo. Interestingly, in the assay for functional BMP2, medium collected from hBM-MSCs transduced with Ad5F35BMP2 stimulated alkaline phosphatase to a level of approximately 5000 chemiluminescence units, whereas the same cells transduced with Ad5BMP2 in the presence of GeneJammer elevated alkaline phosphatase to a level of 9000 chemiluminescence units, demonstrating that this universal system could actually lead to increased BMP2 activity as compared with a parallel system using the chimeric Ad5F35BMP2 (Olmsted-Davis et al., 2002). As expected, this high-level expression of BMP2 after transduction of hBMMSCs with Ad5BMP2 in the presence of GeneJammer led to significant in vivo bone formation after implantation, whereas the cells transduced in parallel in the absence of GeneJammer did not induce detectable mineralization or osteoid by histology. Again, this verifies our previous finding (Gugala et al., 2003) that the level of BMP2 expression dictates the formation of bone and is linked to the efficiency of transduction of the cells. Hence developing a system that would routinely provide high-level transduction of cells with adenovirus, independent of vector type, is an essential component in developing a gene therapy system to induce bone formation. Further, the data also demonstrate that there are no apparent deleterious effects on these processes or the BMP2 protein itself as a result of the potential presence of residual compound in the medium or to the animals after injection of these transduced cells, suggesting that this is safe for conducting preclinical trials.

We next determined the effects of this compound on the efficiency of Ad5BMP2 transduction of a murine cell line, MC3T3-E1, by measuring BMP2 activity in the culture supernatant, using the W20-17 assay. Previous attempts to achieve high-level transduction of this cell line have failed, partly because of the lack of adenovirus-specific receptors for either the Ad5 or Ad5F35 vector (Mallam et al., 2004). However, use of this or other syngeneic murine cells is critical in preclinical studies using Ad5BMP2-transduced cells, because it is derived from the C57BL/6 strain and does not elicit a graft-versus-host reaction in recipient $\mathrm{C} 57 \mathrm{BL} / 6$ mice (data not shown). The results demonstrate that GeneJammer provides a similar enhancement of expression in these murine cells as was observed in hBM-MSCs. Interestingly, at $2500 \mathrm{VP} /$ cell neither transduc- 
tion methodology led to detectable levels of BMP2 protein in the culture supernatant, presumably because of potential lowlevel transduction of the MC3T3-E1 cells. However, at the higher doses of Ad5BMP2 we did observe significant increases in alkaline phosphatase activity correlating with BMP2 activity in the culture supernatant, presumably because of increased transduction of the MC3T3-E1 cells in the presence of GeneJammer. As can be seen in Fig. 4A, culture supernatant taken from MC3T3-E1 cells transduced with Ad5BMP2 (5000 or $10,000 \mathrm{VP} /$ cell) in the presence of GeneJammer led to at least the same or higher levels of alkaline phosphatase and/or BMP2 activity than was achieved with culture supernatant collected from Ad5F35BMP2 (2500 VP/cell)-transduced hBM-MSCs (Fig. 3B), which consistently produced the level of BMP2 necessary to induce bone formation in vivo. These results suggest that the cells are transduced to express BMP2 at a level capable of eliciting bone formation in immune-incompetent mice. The lowest level of BMP2 production we have used to successfully form bone in vivo in immune-incompetent and immune-competent mice are 9 and $18 \mathrm{ng}$ of BMP2 per $10^{6}$ cells per day, respectively. As can be seen in Fig. 4B, when MC3T3E1 cells transduced with Ad5BMP2 (5000 VP/cell) in the presence of GeneJammer were implanted in vivo into C57BL/6 mice, we observed significant bone formation, suggesting that this level of BMP2 is also sufficient to induce bone formation in immunocompetent mice. However, when samples transduced in parallel but in the absence of GeneJammer were implanted we did not observe any bone formation, again presumably because of the low level of BMP2 expression from these cells. Further, these results suggest that high-level BMP2 production is essential regardless of the immune profile of the mice. Interestingly, this is contradictory to a previous report that suggests that low-level virus transduction of cells with potent osteoinductive agents may prevent significant immune response, allowing longer transgene expression and greater bone induction (Kim et al., 2003). In our system the BMP2-transduced cells are eliminated within 4 days yet we still observed robust bone formation, suggesting that avoidance of the immune system is not essential.

We conclude that the use of GeneJammer as described in this study can significantly enhance adenovirus entry into cells even in those lacking adenovirus-specific receptors. Thus this technique provides investigators the ability to transduce a wide range of cells by a rapid, inexpensive method, offering substantial versatility that was previously lacking. Such advances are critical in the translation of cell-based gene therapy systems from small animal models into preclinical and clinical testing.

\section{REFERENCES}

BALAKIREVA, L., SCHOEHN, G., THOUVENIN, E., and CHROBOCZEK, J. (2003). Binding of adenovirus capsid to dipalmitoyl phosphatidylcholine provides a novel pathway for virus entry. J. Virol. 77, 4858-4866.

BERGELSON, J.M., CUNNINGHAM, J.A., DROGUETT, G., KURTJONES, E.A., KRITHIVAS, A., HONG, J.S., HORWITZ, M.S., CROWELL, R.L., and FINBERG, R.W. (1997). Isolation of a common receptor for coxsackie B viruses and adenoviruses 2 and 5. Science 275, 1320-1323.
BLUM, J.S., LI, R.H., MIKOS, A.G., and BARRY, M.A. (2001). An optimized method for the chemiluminescent detection of alkaline phosphatase levels during osteodifferentiation by bone morphogenetic protein 2. J. Cell. Biochem. 80, 532-537.

BONADIO, J., SMILEY, E., PATIL, P., and GOLDSTEIN, S. (1999). Localized, direct plasmid gene delivery in vivo: Prolonged therapy results in reproducible tissue regeneration. Nat. Med. 5, 753-759.

BYK, T., HADDADA, H., VAINCHENKER, W., and LOUACHE, F. (1998). Lipofectamine and related cationic lipids strongly improve adenoviral infection efficiency of primitive human hematopoietic cells. Hum. Gene Ther. 9, 2493-2502.

DAVISON, E., KIRBY, I., ELLIOTT, T., and SANTIS, G. (1999). The human $H L A-A^{*} 0201$ allele, expressed in hamster cells, is not a highaffinity receptor for adenovirus type 5 fiber. J. Virol. 73, 4513-4517.

FRANCESCHI, R.T., YANG, S., RUTHERFORD, R.B., KREBSBACH, P.H., ZHAO, M., and WANG, D. (2004). Gene therapy approaches for bone regeneration. Cells Tissues Organs 176, 95-108. GAGGAR, A., SHAYAKHMETOV, D.M., and LIEBER, A. (2003). CD46 is a cellular receptor for group B adenoviruses. Nat. Med. 9, $1408-1412$.

GAO, W., ROBBINS, P.D., and GAMBOTTO, A. (2003). Human adenovirus type 35: Nucleotide sequence and vector development. Gene Ther. 10, 1941-1949.

GUGALA, Z., OLMSTED-DAVIS, E.A., GANNON, F.H., LINDSEY, R.W., and DAVIS, A.R. (2003). Osteoinduction by ex vivo adenovirus-mediated BMP2 delivery is independent of cell type. Gene Ther. 10, 1289-1296.

HARRISON, G.S., WANG, Y., TOMCZAK, J., HOGAN, C., SHPALL, E.J., CURIEL, T.J., and FELGNER, P.L. (1995). Optimization of gene transfer using cationic lipids in cell lines and primary human $\mathrm{CD}^{+}$and $\mathrm{CD} 34^{+}$hematopoietic cells. Biotechniques 19, 816-823.

HIDAKA, C., MILANO, E., LEOPOLD, P.L., BERGELSON, J.M., HACKETT, N.R., FINBERG, R.W., WICKHAM, T.J., KOVESDI, I., ROELVINK, P., and CRYSTAL, R.G. (1999). CAR-dependent and CAR-independent pathways of adenovirus vector-mediated gene transfer and expression in human fibroblasts. J. Clin. Invest. 103, 579-587.

JAISWAL, N., HAYNESWORTH, S.E., CAPLAN, A.I., and BRUDER, S.P. (1997). Osteogenic differentiation of purified, culture-expanded human mesenchymal stem cells in vitro. J. Cell. Biochem. 64, 295-312.

KIM, H.S., VIGGESWARAPU, M., BODEN, S.D., LIU, Y., HAIR, G.A., LOUIS-UGBO, J., MURAKAMI, H., MINAMIDE, A., SUH, D.Y., and TITUS, L. (2003). Overcoming the immune response to permit ex vivo gene therapy for spine fusion with human type 5 adenoviral delivery of the LIM mineralization protein-1 cDNA. Spine 28, 219-226.

LANUTI, M., KOURI, C.E., FORCE, S., CHANG, M., AMIN, K., XU, K., BLAIR, I., KAISER, L., and ALBELDA, S. (1999). Use of protamine to augment adenovirus-mediated cancer gene therapy. Gene Ther. 6, 1600-1610.

MALLAM, J.N., HURWITZ, M.Y., MAHONEY, T., CHEVEZ-BARRIOS, P., and HURWITZ, R.L. (2004). Efficient gene transfer into retinal cells using adenoviral vectors: Dependence on receptor expression. Invest. Ophthalmol. Vis. Sci. 45, 1680-1687.

MARIT, G., CAO, Y., FROUSSARD, P., RIPOCHE, J., DUPOUY, M., ELANDALOUSSI, A., LACOMBE, F., MAHON, F.X., KELLER, H., PLA, M., REIFFERS, J., and THEZE, J. (2000). Increased liposome-mediated gene transfer into haematopoietic cells grown in adhesion to stromal or fibroblast cell line monolayers. Eur. J. Haematol. 64, 22-31.

MIZUGUCHI, H., and HAYAKAWA, T. (2002). Adenovirus vectors containing chimeric type 5 and type 35 fiber proteins exhibit altered and expanded tropism and increase the size limit of foreign genes. Gene 285, 69-77. 
MUSGRAVE, D.S., BOSCH, P., LEE, J.Y., PELINKOVIC, D., GHIVIZZANI, S.C., WHALEN, J., NIYIBIZI, C., and HUARD, J. (2000). Ex vivo gene therapy to produce bone using different cell types. Clin. Orthop. Relat. Res. 378, 290-305.

OLMSTED, E.A., BLUM, J.S., RILL, D., YOTNDA, P., GUGALA, Z., LINDSEY, R.W., and DAVIS, A.R. (2001). Adenovirus-mediated BMP2 expression in human bone marrow stromal cells. J. Cell. Biochem. 82, 11-21.

OLMSTED-DAVIS, E.A., GUGALA, Z., GANNON, F.H., YOTNDA, P., MCALHANY, R.E., LINDSEY, R.W., and DAVIS, A.R. (2002). Use of a chimeric adenovirus vector enhances BMP2 production and bone formation. Hum. Gene Ther. 13, 1337-1347.

PARK, J., RIES, J., GELSE, K., KLOSS, F., VON DER MARK, K., WILTFANG, J., NEUKAM, F.W., and SCHNEIDER, H. (2003). Bone regeneration in critical size defects by cell-mediated BMP-2 gene transfer: A comparison of adenoviral vectors and liposomes. Gene Ther. 10, 1089-1098.

SAKURAI, F., MIZUGUCHI, H., and HAYAKAWA, T. (2003). Efficient gene transfer into human $\mathrm{CD} 34^{+}$cells by an adenovirus type 35 vector. Gene Ther. 10, 1041-1048.

SEGERMAN, A., ATKINSON, J.P., MARTTILA, M., DENNERQUIST, V., WADELL, G., and ARNBERG, N. (2003). Adenovirus type 11 uses CD46 as a cellular receptor. J. Virol. 77, 9183-9191.

THIES, R.S., BAUDUY, M., ASHTON, B.A., KURTZBERG, L., WOZNEY, J.M., and ROSEN, V. (1992). Recombinant human bone morphogenetic protein-2 induces osteoblastic differentiation in W20-17 stromal cells. Endocrinology 130, 1318-1324.

TOYODA, K., NAKANE, H., and HEISTAD, D.D. (2001). Cationic polymer and lipids augment adenovirus-mediated gene transfer to cerebral arteries in vivo. J. Cereb. Blood Flow Metab. 21, 1125-1131.

TSUDA, H., WADA, T., ITO, Y., UCHIDA, H., DEHARI, H., NAKAMURA, K., SASAKI, K., KOBUNE, M., YAMASHITA, T., and
HAMADA, H. (2003). Efficient BMP2 gene transfer and bone formation of mesenchymal stem cells by a fiber-mutant adenoviral vector. Mol. Ther. 7, 354-365.

TURGEMAN, G., ZILBERMAN, Y., ZHOU, S., KELLY, P., MOUTSATSOS, I.K., KHARODE, Y.P., BORELLA, L.E., BEX, F.J., KOMM, B.S., BODINE, P.V., and GAZIT, D. (2002). Systemically administered rhBMP-2 promotes MSC activity and reverses bone and cartilage loss in osteopenic mice. J. Cell. Biochem. 86, 461-474.

YOTNDA, P., CHEN, D.H., CHIU, W., PIEDRA, P.A., DAVIS, A., TEMPLETON, N.S., and BRENNER, M.K. (2002). Bilamellar cationic liposomes protect adenovectors from preexisting humoral immune responses. Mol. Ther. 5, 233-241.

ZHU, W., RAWLINS, B.A., BOACHIE-ADJEI, O., MYERS, E.R., ARIMIZU, J., CHOI, E., LIEBERMAN, J.R., CRYSTAL, R.G., and HIDAKA, C. (2004). Combined bone morphogenetic protein-2 and -7 gene transfer enhances osteoblastic differentiation and spine fusion in a rodent model. J. Bone Miner. Res. 19, 2021-2032.

Address reprint requests to: Dr. Elizabeth Olmsted-Davis Department of Pediatrics Baylor College of Medicine Houston, TX 77030

E-mail: edavis@bcm.edu

Received for publication June 29, 2005; accepted after revision August 17, 2005.

Published online: September 30, 2005. 